\title{
A Two-Sided BNS Model for Multicurrency FX Markets
}

\author{
Karl Friedrich Bannör, Matthias Scherer and Thorsten Schulz
}

\begin{abstract}
We present a multivariate jump-diffusion model incorporating stochastic volatility and two-sided jumps for multicurrency FX markets, which is an extension of the univariate $\Gamma$-OU-BNS model introduced by [2]. The model can be considered a multivariate variant of the two-sided $\Gamma$-OU-BNS model (cf. [1]). We discuss FX option pricing and provide a calibration exercise, modeling two FX rates with a common currency by a bivariate model and calibrating the dependence parameters to the implied FX volatility surface.
\end{abstract}

Keywords Barndorff-Nielsen-Shephard model $\cdot$ Stochastic volatility $\cdot$ Multivariate model $\cdot$ Jump-diffusion model $\cdot$ Multicurrency FX markets

\section{Introduction}

For derivatives valuation, the Black-Scholes model, presented in the seminal paper [4], generated a wave of stochastic models for the description of stock-prices. Since the assumptions of the Black-Scholes model (normally distributed log-returns, independent returns) cannot be observed in neither time series of stock-prices nor option markets (implicitly expressed in terms of the volatility surface), several alternative models have been developed trying to overcome these assumptions. Some

\author{
K.F. Bannör \\ Deloitte \& Touche GmbH Wirtschaftsprüfungsgesellschaft, Rosenheimer Platz 4, \\ 81669 München, Germany \\ e-mail: kbannoer@deloitte.de \\ M. Scherer · T. Schulz $(\bowtie)$ \\ Technische Universität München, Parkring 11, 85748 Garching-Hochbrück, Germany \\ e-mail: scherer@tum.de \\ T. Schulz \\ e-mail: t.schulz@tum.de \\ (C) The Author(s) 2015 \\ K. Glau et al. (eds.), Innovations in Quantitative Risk Management, \\ Springer Proceedings in Mathematics \& Statistics 99, \\ DOI 10.1007/978-3-319-09114-3_6
}


models, as, e.g., $[9,23]$ account for stochastic volatility, while others as, e.g., [12, 16] enrich the original Black-Scholes model with jumps. Both approaches have been combined in the models of, e.g., [3, 6]. Another approach combining stochastic volatility and negative jumps in both volatility and asset-price process, employing Lévy subordinator-driven Ornstein-Uhlenbeck processes, is available with the Barndorff-Nielsen-Shephard (BNS) model class, presented in [2] and extended in several papers (e.g. [18]). A multivariate extension of the BNS model class employing matrix subordinators is designed in [20] and pricing in this model is scrutinized in [17]. In the special case of a $\Gamma$-OU-BNS model, a tractable variant of a multivariate BNS model based on subordination of compound Poisson processes was developed by [15]. This model allows for a separate calibration of the single assets (following a univariate $\Gamma$-OU-BNS model) and the dependence structure.

Besides for options on stocks, these models have also been used to price derivatives on other underlyings. When modeling foreign exchange (FX) rates instead of stockprices, one has to cope with the introduction of two different interest rates as well as identifying the actual tradeable assets. The Black-Scholes model was adapted to FX markets by [8]. Many of the models mentioned above have been employed for FX rates modeling as, e.g., [3, 9]. Since the original BNS model assumes only downward jumps in the asset-price process, [1] extend the BNS model class to additionally incorporate positive jumps, which is needed for the realistic modeling of FX rates and calibrates much better to FX option surfaces.

In this paper, we unify the extensions of the BNS model from [1, 15] and introduce a multivariate $\Gamma$-OU-BNS model with time-changed compound Poisson drivers incorporating dependent jumps in both directions, both generalizing the univariate two-sided $\Gamma$-OU-BNS model and the multivariate "classical" $\Gamma$-OU-BNS model. Since the two-sided $\Gamma$-OU-BNS model seems to be particularly suitable for the modeling of FX rates, we consider a multivariate two-sided $\Gamma$-OU-BNS model a sensible choice for the valuation of multivariate FX derivatives such as best-of-two options. Since the multivariate two-sided model accounts for joint and single jumps in the FX rates, the jump behavior of modeled FX rates resembles reality better than models only employing joint or single jumps, as illustrated in Fig. 1. Furthermore, a multivariate two-sided BNS model for FX rates with a common currency also implies a jump-diffusion model for an FX rate via quotient or product processes. A crucial feature of our multivariate approach is the separability of the univariate models from the dependence structure, i.e. one has two sets of parameters that can be determined in consecutive steps: parameters determining each univariate model and parameters determining the dependence. This feature provides tractability for practical applications like simulation or calibration on the one side, but also simplifies interpretability of the model parameters on the other side.

Instead of modeling the FX spot rates only, one could model FX forward rates to get a model setup suited for pricing cross-currency derivatives depending on FX forward rates, as for example cross-currency swaps. Multicurrency models built upon FX forward rates (see e.g. [7]) on the one hand support flexibility to price such derivatives, on the other hand, however, these models do not provide the crucial 

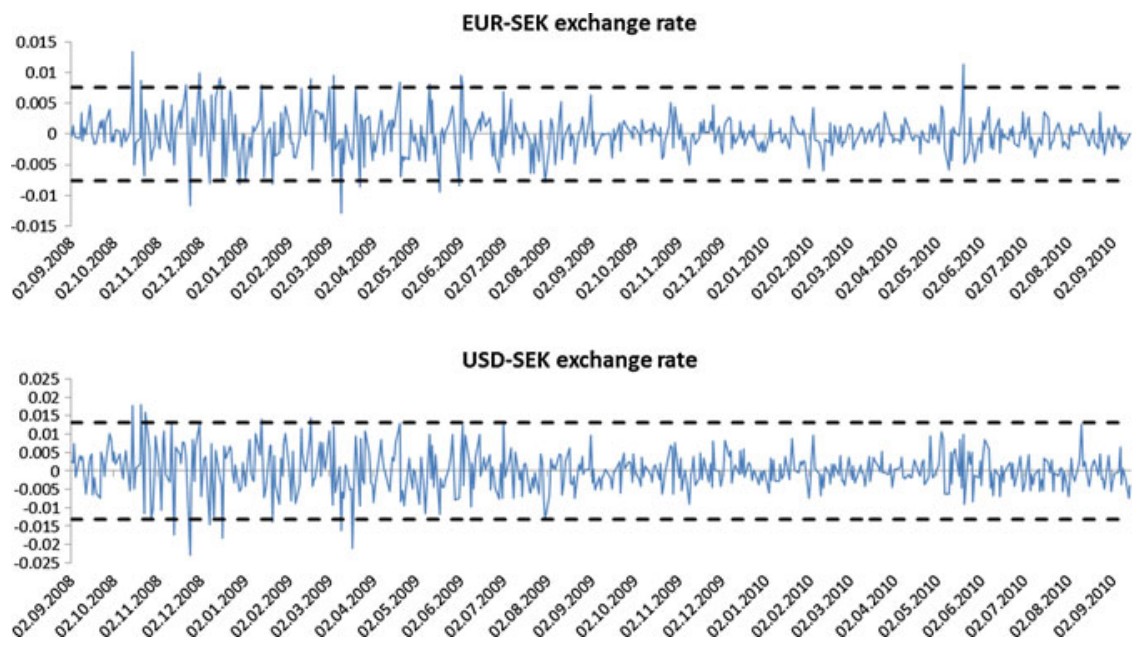

Fig. 1 The logarithmic returns of EUR-SEK and USD-SEK FX rates over time. Assuming that every logarithmic return exceeding three standard deviations (dashed lines) from the mean can be interpreted as a jump (obviously, smaller jumps occur as well, but may be indistinguishable from movement originating in the Brownian noise), one can see that joint as well as separate jumps in the EUR-SEK and the USD-SEK logarithmic returns occur. Clearly, this 3-standard deviation criterion is just a rule of thumb, however, [10] investigated the necessity of both common and individual jumps in a statistical thoroughly manner. Hence, a multivariate FX model capturing the stylized facts of both joint and separate jumps can be valuable. The data was provided by Thomson Reuters

property of separating the dependence structure from the univariate models, which makes it extremely difficult to calibrate such a multivariate model in a sound manner.

The remaining paper is organized as follows: In Sect.2, we recall the two-sided Barndorff-Nielsen-Shephard model constructed in [1] and outline stylized facts of its trajectories. In Sect. 3, we introduce a multivariate version of the two-sided $\Gamma$-OUBNS model, using the time change construction from [15] to incorporate dependence between the jump drivers. Section 4 focuses on the specific obstacles occuring when modeling FX rates in a multivariate two-sided $\Gamma$-OU-BNS model, particularly the dependence structure of joint jumps and the implied model for a third FX rate which may be induced. In Sect. 5, we describe a calibration of the model to implied volatility surfaces and show how the model can be used to price multivariate derivatives. We then evaluate the model in a numerical case study. Finally, Sect. 6 concludes.

\section{The Two-Sided Barndorff-Nielsen-Shephard Model Class}

We briefly motivate the construction and main features of the two-sided BNS model class. The classical BNS model accounts for the leverage effect, a feature of stock returns, by incorporating negative jumps in the asset-price process, accompanied by 
upward jumps in the stochastic variance. While downward jumps might be sufficient in the case of modeling stock-price dynamics, it is not suitable when modeling FX rates, where one-sided jumps contradict economic intuition. Hence, [1] develop an extension of the BNS model which allows for two-sided jumps and is able to capture the symmetric nature of FX rates.

We say that a stochastic process $\left\{S_{t}\right\}_{t \geq 0}$ follows a two-sided BNS model (abbreviated $B N S 2$ model), if the $\log$-price $X_{t}:=\log S_{t}$ follows the dynamics of the SDEs

$$
\begin{aligned}
& \mathrm{d} X_{t}=\left(\mu+\beta \sigma_{t}^{2}\right) \mathrm{d} t+\sigma_{t} \mathrm{~d} W_{t}+\rho_{+} \mathrm{d} Z_{t}^{+}+\rho_{-} \mathrm{d} Z_{t}^{-}, \\
& \mathrm{d} \sigma_{t}^{2}=-\lambda \sigma_{t}^{2} \mathrm{~d} t+\mathrm{d} Z_{t}^{+}+\mathrm{d} Z_{t}^{-},
\end{aligned}
$$

with independent Lévy subordinators $Z^{+}=\left\{Z_{t}^{+}\right\}_{t \geq 0}$ and $Z^{-}=\left\{Z_{t}^{-}\right\}_{t \geq 0}$ and $W=$ $\left\{W_{t}\right\}_{t \geq 0}$ being a Brownian motion independent of $Z^{+}$and $Z^{-}, \mu \in \mathbb{R}, \lambda>0$, $\rho_{+}>0, \rho_{-}<0 .{ }^{1}$ If the Lévy drivers $Z^{+}, Z^{-}$are independent copies of each other, we call the model a reduced two-sided BNS model. If, additionally, $\rho_{+}=-\rho_{-}$we have a symmetric situation, upward jumps occurring similarly likely as downward jumps. Furthermore, the average absolute jump sizes in the log-prices coincide. Thus, we call the model a symmetric BNS model or SBNS model. In a calibration exercise of [1], the SBNS model produced decent calibration results, while limiting the number of parameters to five.

In contrast to the classical BNS model, the BNS2 model has two independent Lévy subordinators $Z^{+}, Z^{-}$incorporating jumps in the asset-price process in opposite directions, but both accounting for upward jumps in the variance process $\sigma^{2}=$ $\left\{\sigma_{t}^{2}\right\}_{t \geq 0}$. Thus, shocks in the asset-price are always accompanied by upward jumping variance, regardless of the jump direction. Furthermore, the variance process is still a Lévy subordinator driven Ornstein-Uhlenbeck process. As discussed in [1], the symmetric nature of the two-sided BNS model makes it particularly suitable for FX rates modeling and calibrates well to option surfaces on FX rates.

An important example is the special case where the Lévy drivers $Z^{+}, Z^{-}$are compound Poisson processes with exponential jump heights. In this case we call the model a two-sided $\Gamma$-OU-BNS model. The log-price of a two-sided $\Gamma$-OU-BNS model has a closed-form characteristic function (cf. [1]), hence allows for rapid calibration to vanilla prices by means of Fourier-pricing methods as introduced in $[5,21]$. A typical trajectory of the two-sided $\Gamma$-OU-BNS model can be found in Fig. 2. It can clearly be seen that shocks in the FX rate process, e.g. caused by macroeconomic turbulences or unanticipated interest rate movements, cause a sudden rise in volatility. As time goes by without the arrival of new shocks, volatility is calming down again.

\footnotetext{
${ }^{1}$ Compared to the original formulation of the model in [1] and the original BNS model from [18], we do not change the clock of the subordinators to $t \mapsto \lambda t$. This formulation is equivalent and more handy in the upcoming multivariate construction.
} 

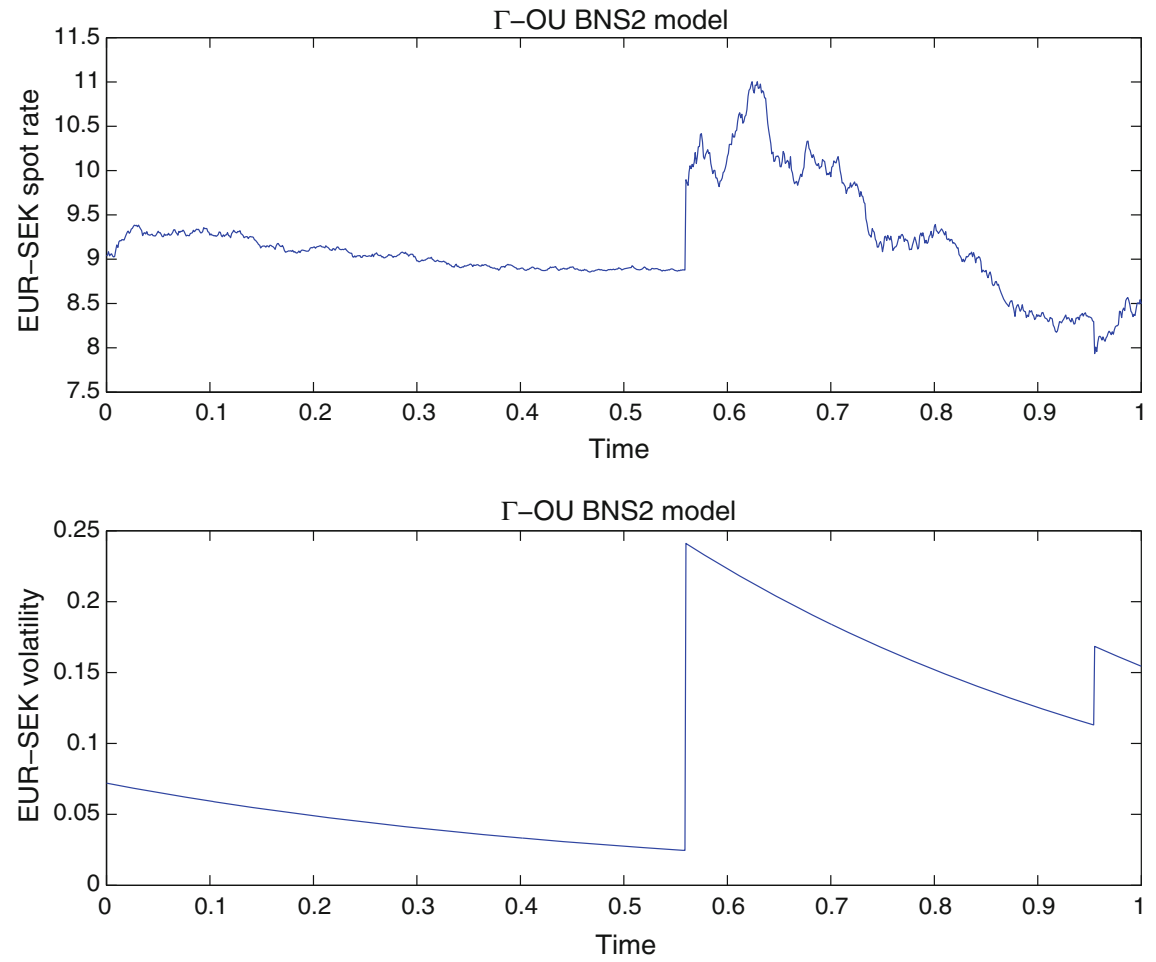

Fig. 2 Sample path of a two-sided BNS model, generated from calibrated parameters. The FX rate process exhibits positive and negative jumps

\section{A Tractable Multivariate Extension of the Two-Sided $\Gamma$-OU-BNS Model}

We now present a multivariate two-sided $\Gamma$-OU-BNS model, where the univariate processes still follow the dynamics of a two-sided $\Gamma$-OU-BNS model. Here, all univariate FX rate processes live on the same probability space and the probability measure is assumed to be a pricing measure. Besides establishing dependence between the driving Brownian motions, we want to incorporate dependence to the Lévy drivers, thus establishing dependence among the price jumps as well as among the variance processes. Jumps in FX rates are mainly driven by unanticipated macroeconomic events (e.g. interest-rate decisions of some central bank) in one of the monetary areas. If we consider a multivariate model with one common currency, e.g. modeling the EUR-USD and the EUR-CHF exchange rates, it is likely that jumps caused by macroeconomic events in the common currency monetary area have an impact on all exchange rates, e.g. the debt crisis of Eurozone countries should affect both the EUR-USD as well as the EUR-CHF exchange rate. Hence, dependence of the jump processes seems to be a desirable feature of a multivariate model for FX 
rates with common currency. To establish dependence between the compound Poisson drivers, we employ the time-change methodology presented in [15], yielding an analytically tractable and easy-to-simulate setup.

Definition 1 (Time-changed CPPs with exponential jump sizes) Let $c_{0}, \eta_{1}, \ldots$, $\eta_{d}>0$ and $c_{1}, \ldots, c_{d} \in\left(0, c_{0}\right)$. Furthermore, let $d \in \mathbb{N}$ and $Y^{(1)}, \ldots, Y^{(d)}$ be $d$ independent compound Poisson processes with intensities $c_{1} /\left(c_{0}-c_{1}\right), \ldots$, $c_{d} /\left(c_{0}-c_{d}\right)$ and $\operatorname{Exp}\left(c_{0} \eta_{1} /\left(c_{0}-c_{1}\right)\right), \ldots, \operatorname{Exp}\left(c_{0} \eta_{d} /\left(c_{0}-c_{d}\right)\right)$-distributed jump sizes. To these compound Poisson processes, we apply a time change with another independent compound Poisson process $T=\left\{T_{t}\right\}_{t \geq 0}$ with $\operatorname{Exp}(1)$-distributed jump sizes and intensity $c_{0}$. Define the $T$-subordinated compound Poisson processes $Z^{(1)}, \ldots, Z^{(d)}$ by $\left\{Z_{t}^{(j)}\right\}_{t \geq 0}:=\left\{Y_{T_{t}}^{(j)}\right\}_{t \geq 0}$. We call the $d$-tuple of $\left(Z^{(1)}, \ldots, Z^{(d)}\right)$ a time-change-dependent multivariate compound Poisson process with parameters $\left(c_{0}, c_{1}, \ldots, c_{d}, \eta_{1}, \ldots, \eta_{d}\right)$.

At first sight, the subordination of a compound Poisson process with another compound Poisson process may look strange, particular in the light of interpreting the time change as "business time", following the idea of [14]. But in this case, we primarily use the joint subordination to introduce dependence via joint jumps between compound Poisson processes without the interpretation as "business time", the time change construction has a technical nature and provides a convenient simulation scheme.

\section{Remark 1 (Properties of time-changed CPPs, cf. [15])}

(i) Each coordinate of the $T$-subordinated compound Poisson process $Z^{(j)}$ is again a compound Poisson process with intensities $c_{j}$ and jump size distribution $\operatorname{Exp}\left(\eta_{j}\right)$ for all $j=1, \ldots, d$.

(ii) For $c_{\max }:=\max _{1 \leq j \leq d}\left\{c_{j}\right\}$, the correlation coefficient of $\left(Z^{(j)}, Z^{(k)}\right), 1 \leq j \leq$ $d, 1 \leq k \leq d, j \neq k$ is given by

$$
\operatorname{Corr}\left[Z_{t}^{(j)}, Z_{t}^{(k)}\right]=\frac{\sqrt{c_{j} c_{k}}}{c_{0}}=\kappa \frac{\sqrt{c_{j} c_{k}}}{c_{\max }},
$$

with $\kappa:=c_{\max } / c_{0} \in(0,1)$. We call $\kappa$ the time-change correlation parameter. In particular, correlation coefficients ranging from zero to $\sqrt{c_{j} c_{k}} / c_{\max }$ are possible, and the correlation does not depend on the point in time $t$.

(iii) Due to the common time change, the compound Poisson processes $Z^{(1)}, \ldots, Z^{(d)}$ are stochastically dependent. Moreover, it can be shown that the dependence structure of the $d$-dimensional process $\left(Z^{(1)}, \ldots, Z^{(d)}\right)$ is driven solely by the time-change correlation parameter $\kappa$.

A striking advantage of introducing dependence among the jumps in this manner is that the time-changed processes $Z^{(1)}, \ldots, Z^{(d)}$ remain in the class of compound Poisson processes with exponential jump heights, which ensures that the marginal processes maintain a tractable structure. In particular, the characteristic functions of the univariate log-price processes in a two-sided $\Gamma$-OU-BNS model are still at 
hand. Moreover, the univariate processes $Z^{(1)}, \ldots, Z^{(d)}$ can be simulated as ordinary compound Poisson processes with exponentially distributed jump heights and the Laplace transform is given. Hence, we can now define a multidimensional two-sided $\Gamma$-OU-BNS model with dependent jumps.

Definition 2 (Multivariate two-sided $\Gamma$-OU-BNS model) A $d$-dimensional stochastic process $\left\{S_{t}\right\}_{t \geq 0}$ with $S_{t}=\left(S_{t}^{(1)}, \ldots, S_{t}^{(d)}\right)$ follows a multivariate two-sided $\Gamma$-OU-BNS model with time-change-dependent volatility drivers, if the dynamics of the $\log$-price vector $X_{t}=\left(X_{t}^{(1)}, \ldots, X_{t}^{(d)}\right)=\left(\log S_{t}^{(1)}, \ldots, \log S_{t}^{(d)}\right)$ are governed by the following SDEs:

$$
\begin{aligned}
\mathrm{d} X_{t}^{(j)} & =\left(\mu_{j}+\beta_{j}\left(\sigma_{t}^{(j)}\right)^{2}\right) \mathrm{d} t+\sigma_{t}^{(j)} \mathrm{d} W_{t}^{(j)}+\rho_{+}^{(j)} \mathrm{d} Z_{t}^{+(j)}+\rho_{-}^{(j)} \mathrm{d} Z_{t}^{-(j)}, \\
\mathrm{d}\left(\sigma_{t}^{(j)}\right)^{2} & =-\lambda_{j}\left(\sigma_{t}^{(j)}\right)^{2} \mathrm{~d} t+\mathrm{d} Z_{t}^{+(j)}+\mathrm{d} Z_{t}^{-(j)}
\end{aligned}
$$

with $\left(W^{(1)}, \ldots, W^{(d)}\right)$ being correlated Brownian motions with correlation matrix $\Sigma$ and for all $1 \leq j \leq d, \mu_{j}, \beta_{j} \in \mathbb{R}, \rho_{+}^{(j)}>0, \rho_{-}^{(j)}<0, \lambda_{j}>0$, and $\left(Z^{+(1)}, Z^{-(1)}\right), \ldots,\left(Z^{+(d)}, Z^{-(d)}\right)$ are pairs of independent compound Poisson processes with exponential jumps. Furthermore, the $2 d$-dimensional Lévy process $\left(Z^{+(1)}, Z^{-(1)}, \ldots, Z^{+(d)}, Z^{-(d)}\right)$ splits up in two time-change-dependent $d$-tuples of compound Poisson processes (cf. Definition 1).

At first glance, Definition 2 looks cumbersome, but it is necessary to capture all combinations of possible dependence. As a simplifying example, one might think about introducing dependence between $\left(Z^{+(1)}, \ldots, Z^{+(d)}\right)$ on the one hand and between $\left(Z^{-(1)}, \ldots, Z^{-(d)}\right)$ on the other hand. In this case, positive jumps of the processes are mutually dependent and negative jumps are mutually dependent, but positive jumps occur independently of negative jumps. A closer examination how to establish the dependence structure between the time-change-dependent compound Poisson processes is made in the following section, since dependence between the jumps has to be introduced in a sound economic manner.

This construction can further be generalized by employing Lévy processes, coupled by Lévy copulas (cf. [11]). For the present investigation, however, we prefer the time-change construction presented in Definition 1, since this construction provides an immediate stochastic representation of the dependence structure. Thus, a straightforward simulation scheme is provided and at least some analytical tractability when doing computational exercises is ensured, which may be more complicated when employing general Lévy copulas.

Remark 2 (Calibration of the univariate processes) An immediate corollary from the compound Poisson structure of the univariate jump processes $\left(Z^{+(j)}, Z^{-(j)}\right)$, $j=1, \ldots, d$, is that the univariate log-price processes $\left\{X_{t}^{(j)}\right\}_{t \geq 0}, j=1, \ldots, d$, still follow a univariate two-sided $\Gamma$-OU-BNS model and the parameters of the univariate processes may be calibrated separately to univariate derivative prices. 
The dependence parameters, which are the correlation matrix $\Sigma$ of the Brownian motions and the time-change correlation parameters $\tilde{\kappa}$ and $\hat{\kappa}$ that determine the dependence structure of the time-change-dependent multivariate compound Poisson processes, can be calibrated separately afterwards without altering the already fixed marginal distributions. This simplifies the model calibration and is a convenient feature for practical purposes, because it automatically ensures that univariate derivative prices are fitted to the multivariate model.

\section{Modeling Two FX Rates with a Bivariate Two-Sided $\Gamma$-OU-BNS Model}

In this section, we discuss the modeling of FX rates with a bivariate two-sided $\Gamma$-OUBNS model. Particularly, we discuss how to soundly introduce dependence between the Lévy drivers and investigate a possible "built-in" model induced by the model for the two FX rates. We concentrate on the case of two currency pairs, which illustrates the problems of choosing the jump dependence structure best.

To ensure familiarity with the FX markets wording, we recall that an FX rate is the exchange rate between two currencies, expressed as a fraction. The currency in the numerator of the fraction is called (by definition) domestic currency, while the currency in the denominator of the fraction is called foreign currency. ${ }^{2}$ The role each currency plays in an FX rate is defined by market conventions and is often due to historic reasons, so economic interpretations are not necessarily helpful. A more detailed discussion of market conventions of FX rates and derivatives is provided in [22], a standard textbook on FX rates modeling is [13].

\subsection{The Dependence Structure of the Lévy Drivers}

Analogously to the multivariate classical $\Gamma$-OU-BNS model described in the previous section, we use the time-change construction to introduce dependence between the compound Poisson drivers in the bivariate two-sided $\Gamma$-OU-BNS model. Since we want to model dependence between the jumps in different FX rates, we have to choose the coupling of the compound Poisson drivers carefully and in a way to capture economic intuition: When modeling two FX rates, we may want to establish an adequate kind of dependence between the different drivers, accounting separately for positive and negative jumps in the respective FX rate. Depending on which currency is foreign or domestic in the two currency pairs of the FX rates, dependence may be

\footnotetext{
2 The wording "foreign" and "domestic" currency does not necessarily reflect whether the currency is foreign or domestic from the point of view of a market participant. The currency EUR, e.g., is always foreign currency by market convention. Sometimes, the foreign currency is called underlying currency, while the domestic currency is called accounting or base currency.
} 
introduced in a different manner to result in sound economic situations. Hence, we can distinguish between the following combinations that may occur for two different FX rates:

1. There are no common currencies, e.g. in the case of EUR-CHF and USD-JPY.

2. In both FX rates the common currency is the foreign (resp. domestic) currency, e.g. EUR-USD and EUR-CHF (EUR-CHF and USD-CHF, respectively).

3. The common currency is the domestic currency in one FX rate and the foreign currency in the other FX rate, e.g. EUR-USD and USD-CHF.

For the sake of simplicity, we restrict ourselves to the second case, which occurs in a detailed numerical study in the following section. The other cases can be treated analoguously.

In case of a common foreign currency, a sudden macroeconomic event strengthening (resp. weakening) the common currency should result in an upward (resp. downward) jump of both FX rates. Hence, it may be a sensible choice to couple the drivers for the positive jumps and to separately couple the drivers for the negative jumps respectively, to ensure the occurrence of joint upward and downward jumps.

\subsection{Implicitly Defined Models}

When two FX rates are modeled and among the two rates there is a common currency, this bivariate model always implicitly defines a model for the missing currency pair which is not modeled directly, e.g. when modeling EUR-USD and EUR-CHF exchange rates simultaneously, the quotient process automatically implies a model for the USD-CHF exchange rate. Similar to the bivariate Garman-Kohlhagen model, modeling two FX rates directly by a bivariate two-sided BNS model does not necessarily imply a similar model for the quotient or product process from the same family, but the main structure of a jump-diffusion-type model is maintained.

Lemma 1 (Quotient and product process of a two-sided BNS model) Given two asset-price processes $\left\{S_{t}^{(1)}\right\}_{t \geq 0}$ and $\left\{S_{t}^{(2)}\right\}_{t \geq 0}$ modeled by a multivariate two-sided $\Gamma$-OU-BNS models, the product and quotient processes $\left\{S_{t}^{(1)} S_{t}^{(2)}\right\}_{t \geq 0}$ resp. $\left\{S_{t}^{(1)} / S_{t}^{(2)}\right\}_{t \geq 0}$ are both of jump-diffusion type.

Proof Follows directly from $\log \left(S_{t}^{(1)} S_{t}^{(2)}\right)=X_{t}^{(1)}+X_{t}^{(2)}$ and $\log \left(S_{t}^{(1)} / S_{t}^{(2)}\right)=$ $X_{t}^{(1)}-X_{t}^{(2)}$.

Due to symmetry in FX rates, the implied model for the third missing FX rate can be used to calibrate the parameters steering the dependence, namely, the correlation between the Brownian motions as well as the time-change correlation parameters, or equivalently the intensities of the time-change processes. Additionally, the calibration performance of the implied model to plain vanilla options yields a plausibility check whether the bivariate model may be useful for the evaluation of true bivariate options, e.g. best-of-two options or spread options. 


\section{Application: Calibration to FX Rates and Pricing of Bivariate FX Derivatives}

In this section, we describe the calibration process of a bivariate two-sided BNS model to market prices of univariate FX derivatives, which allows us to completely specify the model. Furthermore, we describe how to price bivariate FX options like, e.g., best-of-two options in a bivariate two-sided BNS model.

\subsection{Data}

As input data for our calibration exercise we use option data on exchange rates concerning the three currencies EUR, USD, and SEK. Since the EUR-USD exchange rate can be regarded as an implied exchange rate, i.e.

$$
\frac{\mathrm{USD}}{\mathrm{EUR}}=\frac{\mathrm{SEK} / \mathrm{EUR}}{\mathrm{SEK} / \mathrm{USD}}
$$

we model the two exchange rates EUR-SEK and USD-SEK directly with two-sided $\Gamma$-OU-BNS models as suggested in [1]. For each currency pair EUR-SEK, USDSEK, and EUR-USD, we have the implied volatilities of 204 different plain vanilla options (different maturities, different moneyness) available as input data. The option data is as of August 13, 2012, and was provided by Thomson Reuters.

\subsection{Model Setup}

We consider a market with two traded assets, namely $\left\{\exp \left(r_{\mathrm{USD}} t\right) S_{t}^{\mathrm{USDSEK}}\right\}_{t \geq 0}$ and $\left\{\exp \left(r_{\mathrm{EUR}} t\right) S_{t}^{\mathrm{EURSEK}}\right\}_{t \geq 0}$, where $S_{t}^{\mathrm{USDSEK}}, S_{t}^{\mathrm{EURSEK}}$ denote the exchange rates at time $t$ and $r_{\mathrm{USD}}, r_{\mathrm{EUR}}, r_{\mathrm{SEK}}$ denote the risk free interest rates in the corresponding monetary areas. These assets can be seen as the future value of a unit of the respective foreign currency (in this case USD or EUR), valued in the domestic currency (which is SEK). Assume a risk-neutral measure $\mathbb{Q}^{\text {SEK }}$ to be given with numéraire process $\left\{\exp \left(r_{\mathrm{SEK}} t\right)\right\}_{t \geq 0}$, i.e. $\left\{\exp \left(\left(r_{\mathrm{USD}}-r_{\mathrm{SEK}}\right) t\right) S_{t}^{\mathrm{USDSEK}}\right\}_{t \geq 0}$ and $\left\{\exp \left(\left(r_{\mathrm{EUR}}-r_{\mathrm{SEK}}\right) t\right) S_{t}^{\mathrm{EURSEK}}\right\}_{t \geq 0}$ are martingales with respect to $\mathbb{Q}^{\mathrm{SEK}}$, governed by the SDEs 


$$
\begin{aligned}
\mathrm{d} X^{\star \mathrm{SEK}}= & \left(r_{\mathrm{SEK}}-r_{\star}-\frac{\left(\sigma_{t}^{\star \mathrm{SEK}}\right)^{2}}{2}-\frac{c_{\star \mathrm{SEK}}^{+} \rho_{\star \mathrm{SEK}}^{+}}{\eta_{\star \mathrm{SEK}}^{+}-\rho_{\star \mathrm{SEK}}^{+}}+\frac{c_{\star \mathrm{SEK}}^{-} \rho_{\star \mathrm{SEK}}^{-}}{\eta_{\star \mathrm{SEK}}^{-}+\rho_{\star \mathrm{SEK}}^{-}}\right) \mathrm{d} t \\
& +\sigma_{t}^{\star \mathrm{SEK}} \mathrm{d} W_{t}^{\star \mathrm{SEK}}+\rho_{\star \mathrm{SEK}}^{+} \mathrm{d} Z_{t}^{+\star \mathrm{SEK}}-\rho_{\star \mathrm{SEK}}^{-} \mathrm{d} Z_{t}^{-\star \mathrm{SEK}}, \\
\mathrm{d} \sigma_{t}^{2 \star \mathrm{SEK}}= & -\lambda_{\star} \mathrm{SEK} \sigma_{t}^{2 \star \mathrm{SEK}} \mathrm{d} t+\mathrm{d} Z_{t}^{+\star \mathrm{SEK}}+\mathrm{d} Z_{t}^{-\star \mathrm{SEK}},
\end{aligned}
$$

for $\lambda_{\star \text { SEK }}, \rho_{\star \text { SEK }}^{+}, \rho_{\star \text { SEK }}^{-}>0, \star \in\{$ EUR, USD $\},\left\{W_{t}^{\text {EURSEK }}, W_{t}^{\text {USDSEK }}\right\}_{t \geq 0}$ being a two-dimensional Brownian motion with correlation $r \in[-1,1]$, and $\left\{Z_{t}^{+}\right.$EURSEK

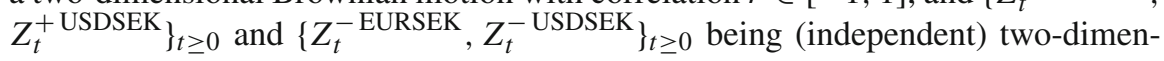
sional time-change dependent compound Poisson processes with parameters

$$
\begin{array}{r}
\left(\max \left(c_{\mathrm{EURSEK}}^{+}, c_{\mathrm{USDSEK}}^{+}\right) / \kappa^{+}, c_{\mathrm{EURSEK}}^{+}, c_{\mathrm{USDSEK}}^{+}, \eta_{\mathrm{EURSEK}}^{+}, \eta_{\mathrm{USDSEK}}^{+}\right) \\
\text {and }\left(\max \left(c_{\mathrm{EURSEK}}^{-}, c_{\mathrm{USDSEK}}^{-}\right) / \kappa^{-}, c_{\mathrm{EURSEK}}^{-}, c_{\mathrm{USDSEK}}^{-}, \eta_{\mathrm{EURSEK}}^{-}, \eta_{\mathrm{USDSEK}}^{-}\right),
\end{array}
$$

where $\kappa^{+}$and $\kappa^{-}$are the time-change correlation parameters (following the framework in Sect. 4.1). Hence, the EUR-SEK, EUR-USD exchange rates follow a bivariate SBNS model. The implied exchange rate process $S^{\text {EURUSD }}$ is given by

$$
\left\{S_{t}^{\text {EURUSD }}\right\}_{t \geq 0}=\left\{\frac{S_{t}^{\text {EURSEK }}}{S_{t}^{\text {USDSEK }}}\right\}_{t \geq 0} .
$$

Due to the change-of-numéraire formula for exchange rates (cf. [19]), the process $\left\{\exp \left(\left(r_{\text {EUR }}-r_{\mathrm{USD}}\right) t\right) S_{t}^{\mathrm{EURUSD}}\right\}_{t \geq 0}$ is a martingale with respect to $\mathbb{Q}^{\mathrm{USD}}$, where $\mathbb{Q}^{\text {USD }}$ is determined by the Radon-Nikodým derivative

$$
\left.\frac{\mathrm{d} \mathbb{Q}^{\mathrm{USD}}}{\mathrm{d} \mathbb{Q}^{\mathrm{SEK}}}\right|_{t}=\frac{S_{t}^{\mathrm{USDSEK}} \exp \left(r_{\mathrm{USD}} t\right)}{S_{0}^{\mathrm{USDSEK}} \exp \left(r_{\mathrm{SEK}} t\right)} .
$$

\subsection{Calibration}

For calibration purposes, we use the volatility surfaces of the EUR-SEK and USDSEK exchange rates to fit the univariate parameters. Due to the consistency relationships which have to hold between the exchange rates, we can calibrate the dependence parameters by fitting them to the volatility surface of EUR-USD. Even in presence of other "bivariate options" (e.g. best-of-two options), we argue that European options on the quotient exchange rate currently provide the most liquid and reliable data for a calibration.

The calibration of the presented multivariate model is done in two steps. Due to the fact that the marginal distributions can be separated from the dependence structure within our models, it is possible to keep the parameters governing the dependence 
Table 1 Calibrated parameters in the two univariate FX models

\begin{tabular}{l|l|l|l|l|l|l|l|l}
\hline$\star$ & $S_{0}^{\star \text { SEK }}$ & $\sigma_{0}^{\star \text { SEK }}$ & $c_{\star \text { SEK }}$ & $\eta_{\star \text { SEK }}$ & $\lambda_{\star}$ SEK & $\rho_{\star \text { SEK }}$ & \#options & Error $(\%)$ \\
\hline EUR & 8.229 & 0.074 & 0.71 & 62.13 & 3.25 & 1.66 & 204 & 1.08 \\
\hline USD & 6.664 & 0.078 & 1.15 & 40.81 & 2.19 & 1.22 & 204 & 3.17 \\
\hline
\end{tabular}

separated from the parameters governing the marginal distributions. Therefore, in a first step we independently calibrate both univariate models for the EUR-SEK and USD-SEK exchange rates, and in a second step we calibrate the parameters driving the dependence structure. In doing so, the fixed univariate parameters are not affected by the second step. Since there is little market data of multi-currency options, this two step method is very appealing: we can disintegrate one big calibration problem in two smaller ones. The univariate models are calibrated to volatility surfaces of the EURSEK and USD-SEK exchange rates via minimizing the relative distance of the model implied option prices to market prices, with equal weight on every option. Option prices in the univariate two-sided BNS models are obtained via Fourier inversion (cf. $[5,21])$ by means of the characteristic function of the log-prices.

Table 1 gives an overview of the calibration result of the univariate models. To reduce the number of parameters, we use symmetric two-sided $\Gamma$-OU-BNS models as described in [1]. Furthermore, we assume that the time-change correlation parameters $\kappa^{+}$and $\kappa^{-}$coincide; maintaining the symmetric structure. The relative error in model prices with respect to market prices of the 204 options can be seen as calibration error. The average relative error in the EUR-SEK-model is about one percent, and in the USD-SEK-model it is around three percent. Hence, the univariate models fit the FX market reasonably well. Each univariate calibration requires about $20 \mathrm{~s}$.

The calibration of the parameters governing the dependence is done by means of the third implied exchange rate, namely by the volatility surface of EUR-USD. Model prices of EUR-USD-options with payout function $f$ at time $t$ can be obtained by a Monte-Carlo simulation of the following expected value:

$$
\begin{aligned}
& \mathbb{E}_{\mathbb{Q}} \text { USD }\left[f\left(S_{t}^{\text {EURUSD }}\right) \exp \left(-r_{\text {USD }} t\right)\right] \\
& \quad=\mathbb{E}_{\mathbb{Q}^{\text {SEK }}}\left[f\left(\frac{S_{t}^{\text {EURSEK }}}{S_{t}^{\text {USDSEK }}}\right) \frac{S_{t}^{\text {USDSEK }}}{S_{0}^{\text {USDSEK }}} \exp \left(-r_{\text {SEK }} t\right)\right]
\end{aligned}
$$

Here, we used 100,000 simulations to calibrate the dependence parameters. The execution of the overall optimization procedure takes around four hours. The calibration error of the dependence parameters in terms of average relative error is roughly nine percent, which is still a good result giving consideration to the fact that we try to fit 204 market prices by means of just two parameters in an implicitly specified model. A more complex model, obtained by relaxing the condition that $\kappa^{+}$and $\kappa^{-}$ 


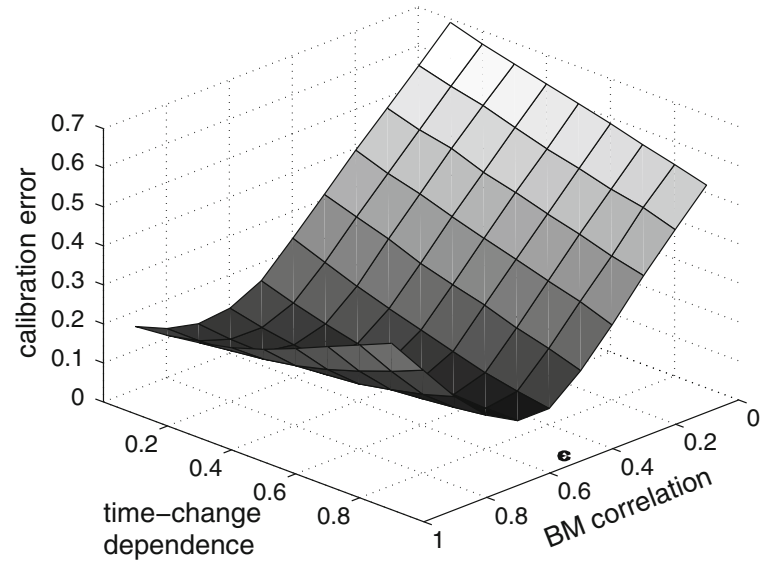

Fig. 3 The best matching correlation between the two Brownian motions is 0.52 and the optimal time-change dependence parameter is $\kappa=0.96$. This corresponds to a calibration error of around nine percent for the 204 options on this currency pair

coincide, leads to even smaller calibration errors. However, we keep the model as simple as possible to maintain tractability. Figure 3 illustrates the calibration error of this second step depending on different choices of the dependence parameters. Eventually, the whole model is fixed.

Now, we are able to price European multi-currency options, for instance a bestof-two call option with a payoff at time $t$ given by

$$
\max \left\{\max \left\{\frac{e^{X_{T}^{\text {USDSEK }}}}{S_{0}^{\text {USDSEK }}}-K, 0\right\}, \max \left\{\frac{e^{X_{T}^{\text {EURSEK }}}}{S_{0}^{\text {EURSEK }}}-K, 0\right\}\right\},
$$

i.e. we consider the maximum of two call options with strike $K>0$ on two exchange rates. This option can be used as an insurance against a weakening SEK, because one gets a payoff if the relative performance of one exchange rate, USD-SEK or EUR$\mathrm{SEK}$, is greater than $K-1$. Pricing is done by a Monte-Carlo simulation that estimates the expected value in Eq. (1). We used 100,000 scenarios to price this option, which takes about four minutes. Figure 4 shows option prices of the best-of-two call option dependent on various choices of the dependence parameters. 


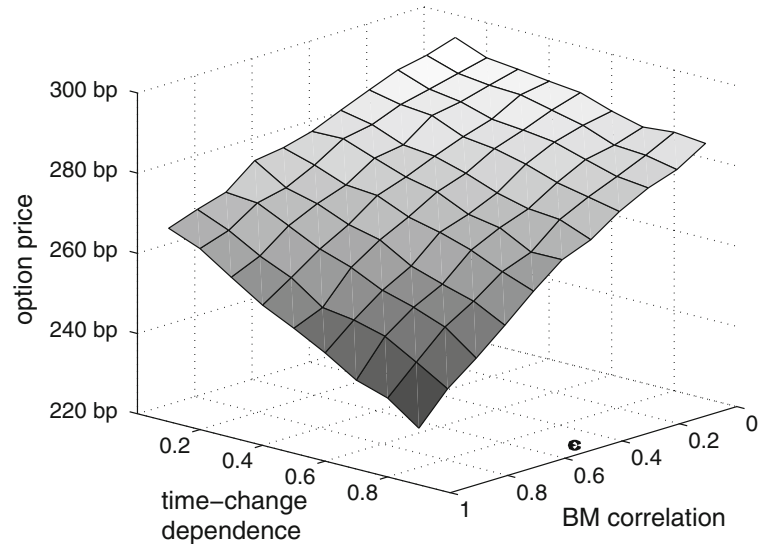

Fig. 4 Prices of a best-of-two call option where $K=1.1$ and $T=1$. One observes that both dependence parameters play an important role for the price of this option. For the optimal parameter setting (Brownian motion correlation is $0.52, \kappa=0.96$ ), the fair price of this option is $261 \mathrm{bp}$

\section{Conclusion and Outlook}

We introduced a multi-dimensional FX rate model generalizing the univariate twosided BNS model in a way that each FX rate is still modeled as a two-sided BNS model. Thus, the parameters driving the dependence structure can be separated from the marginal distributions. This simplifies the calibration of the overall model tremendously, such that the multicurrency model can be calibrated to plain vanilla FX option prices. As an outlook for further research, we wonder whether there exists a measure change from the real world measure to the martingale measure we assumed to exist in the first place.

Acknowledgments We thank the KPMG Center of Excellence in Risk Management for making this work possible. Furthermore, we thank the TUM Graduate School for supporting these studies and two anonymous referees for valuable comments.

Open Access This chapter is distributed under the terms of the Creative Commons Attribution Noncommercial License, which permits any noncommercial use, distribution, and reproduction in any medium, provided the original author(s) and source are credited.

\section{References}

1. Bannör, K., Scherer, M.: A BNS-type stochastic volatility model with two-sided jumps, with applications to FX options pricing. Wilmott 2013(65), 58-69 (2013)

2. Barndorff-Nielsen, O.E., Shephard, N.: Non-Gaussian Ornstein-Uhlenbeck-based models and some of their uses in financial economics. J. R. Stat. Soc.: Ser. B (Stat. Methodol.) 63, 167-241 (2001) 
3. Bates, D.: Jumps and stochastic volatility: exchange rate processes implicit in Deutsche Mark options. Rev. Financ. Stud. 9(1), 69-107 (1996)

4. Black, F., Scholes, M.: The pricing of options and corporate liabilities. J. Polit. Econ. 81(3), 637-654 (1973)

5. Carr, P., Madan, D.: Option valuation using the fast Fourier transform. J. Comput. Financ. 2, 61-73 (1999)

6. Duffie, D., Pan, J., Singleton, K.: Transform analysis and asset pricing for affine jumpdiffusions. Econometrica 68, 1343-1376 (2000)

7. Eberlein, E., Koval, N.: A cross-currency Lévy market model. Quant. Financ. 6(6), 465-480 (2006)

8. Garman, M., Kohlhagen, S.: Foreign currency option values. J. Int. Money Financ. 2, 231-237 (1983)

9. Heston, S.: A closed-form solution for options with stochastic volatility with applications to bond and currency options. Rev. Financ. Stud. 6(2), 327-343 (1993)

10. Jacod, J., Todorov, V.: Testing for common arrivals of jumps for discretely observed multidimensional processes. Ann. Stat. 37(4), 1792-1838 (2009)

11. Kallsen, J., Tankov, P.: Characterization of dependence of multidimensional Lévy processes using Lévy copulas. J. Multivar. Anal. 97(7), 1551-1572 (2006)

12. Kou, S.G.: A jump-diffusion model for option pricing. Manag. Sci. 48(8), 1086-1101 (2002)

13. Lipton, A.: Mathematical Methods For Foreign Exchange: A Financial Engineer's Approach. World Scientific Publishing Company, River Edge (2001)

14. Luciano, E., Schoutens, W.: A multivariate jump-driven financial asset model. Quant. Financ. 6(5), 385-402 (2006)

15. Mai, J.-F., Scherer, M., Schulz, T.: Sequential modeling of dependent jump processes. Wilmott 2014(70), 54-63 (2014)

16. Merton, R.: Option pricing when underlying stock returns are discontinuous. J. Financ. Econ. 3(1), 125-144 (1976)

17. Muhle-Karbe, J., Pfaffel, O., Stelzer, R.: Option pricing in multivariate stochastic volatility models of OU type. SIAM J. Financ. Math. 3, 66-94 (2012)

18. Nicolato, E., Venardos, E.: Option pricing in stochastic volatility models of the OrnsteinUhlenbeck type. Math. Financ. 13(4), 445-466 (2003)

19. Pelsser, A.: Mathematical foundation of convexity correction. Quant. Financ. 3(1), 59-65 (2003)

20. Pigorsch, C., Stelzer, R.: A multivariate generalization of the Ornstein-Uhlenbeck stochastic volatility model, working paper (2008)

21. Raible, S.: Lévy Processes in finance: theory, numerics, and empirical facts, $\mathrm{PhD}$ thesis, Albert-Ludwigs-Universität Freiburg i. Br. (2000)

22. Reiswich, D., Wystup, U.: FX volatility smile construction. Wilmott 60, 58-69 (2012)

23. Stein, E., Stein, J.: Stock price distributions with stochastic volatility: an analytic approach. Rev. Financ. Stud. 4(4), 727-752 (1991) 